\title{
Anesthesia and perioperative pain management during cardiac electronic device implantation
}

\section{Marina Biocic' \\ Dijana Vidosevic ${ }^{2}$ \\ Matija Boric ${ }^{1,3}$ \\ Teo Boric ${ }^{4}$ \\ Lovel Giunio ${ }^{2}$ \\ Damir Fabijanic ${ }^{2}$ \\ Livia Puljak ${ }^{1,5}$}

'Laboratory for Pain Research, University of Split School of

Medicine, ${ }^{2}$ Department of Cardiology, ${ }^{3}$ Department of Abdominal Surgery,

${ }^{4}$ Department of Vascular Surgery,

University Hospital Split, Split,

${ }^{5}$ Department for Development,

Research and Health Technology

Assessment, Agency for Quality and

Accreditation in Health Care and

Social Welfare, Zagreb, Croatia
Correspondence: Livia Puljak

Laboratory for Pain Research, University of Split School of Medicine, Soltanska 2, 21000 Split, Croatia

$\mathrm{Tel}+38521557807$

Fax+38521557811

Email livia@mefst.hr
This article was published in the following Dove Press journal:

Journal of Pain Research

19 April 2017

Number of times this article has been viewed

Background: The degree of pain caused by the implantation of cardiac electronic devices (CEDs) and the type of anesthesia or perioperative pain management used with the procedure have been insufficiently studied. The aim of this study was to analyze perioperative pain management, as well as intensity and location of pain among patients undergoing implantation of CED, and to compare the practice with published guidelines.

Patients and methods: This was a combined retrospective and prospective study conducted at the tertiary hospital, University Hospital Split, Croatia. The sample included 372 patients who underwent CED implantation. Perioperative pain management was analyzed retrospectively in 321 patients who underwent CED implantation during 2014. In a prospective study, intensity and location of pain before, during, and after the procedure were measured by using a numerical rating scale (NRS) ranging from 0 to 10 in 51 patients at the same institution from November 2014 to August 2015.

Results: A quarter of patients received analgesia or sedation before surgery. All the patients received local lidocaine anesthesia. After surgery, $31 \%$ of patients received pain medication or sedation. The highest pain intensity was observed during CED implantation with the highest NRS pain score being 8 . Some patients reported severe pain (NRS >5) also at 1, 3, 6, 8, and 24 hours after surgery. The most common pain locations were surgical site, shoulder, and chest. Adherence to guidelines for acute perioperative pain management was insufficient.

Conclusion: Patients may experience severe pain during and after CED implantation. Perioperative pain management was suboptimal, and higher doses of sedation and intensive analgesia are required. Guidelines for acute perioperative pain management and anesthesia during CED implantation should be developed.

Keywords: cardiac electronic devices, perioperative pain management, postoperative pain, analgesics, pain intensity, guidelines

\section{Introduction}

The use of implantable cardiac electronic devices (CEDs), including pacemakers, implantable cardioverter-defibrillators, and cardiac resynchronized therapy (CRT), is increasing worldwide for the treatment of cardiac arrhythmias. ${ }^{1-3}$

Pain-free life is nowadays considered an essential human right. ${ }^{4}$ However, despite the growing consumption of analgesics worldwide, ${ }^{5-9}$ the prevalence of postoperative pain in various surgical disciplines is worrying. ${ }^{10-12}$ It is known that poorly treated acute perioperative pain may lead to chronic postsurgical pain (CPSP) and that the prevalence of CPSP is also disturbingly high. For example, an Italian study showed that the incidence of CPSP at 6 months was $45.2 \%$ for mild pain, $15.9 \%$ for moderate pain, 
and $2.7 \%$ for severe pain, whereas the incidence of CPSP at 12 months was $35.9 \%, 11.8 \%$, and $2.5 \%$ for mild, moderate, and severe pain, respectively. ${ }^{11}$ Multiple factors may be associated with postoperative pain, ${ }^{13}$ and perioperative pain management is not necessarily conducted according to the recommended international guidelines. ${ }^{14}$

No data have been published to provide evidence about the degree of pain caused by implantation of CEDs, the type of anesthesia used during the procedure, or perioperative pain management. The aim of this study was to explore the patterns of anesthesia and perioperative pain management during CED implantation from patient records and to study pain intensity and location experienced by patients perioperatively in a prospective study.

\section{Patients and methods Design and setting}

This study included both retrospective and prospective data collection. Data on anesthesia and analgesia were collected retrospectively for all patients $(\mathrm{N}=321)$ who underwent surgical cardiac device implantation at the University Hospital Split, Croatia, from January to December 2014. Data on pain intensity and location during CED implantation were collected prospectively from a convenience sample of 51 patients treated between November 2014 and August 2015.

\section{Ethics}

The study was approved by the ethics committee of The University Hospital Split. Patient consent was not required to access their medical records for the retrospective study because all data analyzed in the retrospective study were collected as part of routine diagnosis and treatment. Patients were recruited to the prospective study after receiving detailed information about the study and providing informed consent.

\section{Data collection}

Types of anesthesia, analgesia, and perioperative pain management were analyzed during and after cardiac device therapy and compared to the American Society of Anesthesiologists (ASA) guidelines for perioperative pain management. ${ }^{15}$

The data collected in the retrospective part of the study included age, sex, diagnosis, type and dosage of premedication, type of cardiac device, type and dosage of anesthesia, and type and dosage of analgesia for each day if it was administered on more than 1 day (on each postoperative day, whether the patient received pain medicine, type of analgesia, and dose were noted).
In the prospective part of the study, pain intensity was measured before, during, and after implantation (at 1, 3, 6, and 8 hours after device implantation, and 24 hours after implantation if the patient was still in the hospital). Pain intensity was measured by using a numerical rating scale (NRS) ranging from 0 to 10 , with 0 indicating no pain and 10 indicating the worst pain imaginable. Patients were also asked about the location of their pain. Data about pain intensity and location were recorded by a nurse on a special data collection form, based on the patients' self-report.

\section{Statistics}

Descriptive statistics were performed by using Microsoft Excel (Microsoft Inc., Redmond, WA, USA). Frequencies and percentages were calculated for the analyzed categorical variables. For continuous variables, mean and standard deviation were calculated.

\section{Results}

\section{Retrospective study}

A total of 321 patients, who underwent CED implantation during 2014 at the University Hospital, Split, were included in the retrospective study. They comprised 201 male $(63 \%)$ and 120 female $(37 \%)$ patients with an average age of 76 (range: 24-103) years. The three most common indications for surgery were atrioventricular block, atrial fibrillation, and sinus node dysfunction.

Patients included in the retrospective study received several types of devices: pacemaker (96\%), cardioverterdefibrillator $(2 \%)$, CRT $(1 \%)$, and "loop recorder" $(0.6 \%)$. Local anesthesia with 5-10 mg lidocaine was applied during CED implantation as the only type of anesthesia in all patients. None of the patients received general anesthesia.

Of the 321 patients, 242 (75\%) did not receive any drug before the procedure. Among the $25 \%$ of patients who received premedication, the most commonly used drugs were a combination of tramadol and acetaminophen (28\%), tramadol (25\%), diazepam (20\%), acetaminophen, and ibuprofen $(8 \%)$, whereas a few patients received methylphenobarbital $(2 \%)$, zolpidem $(2 \%)$, diclofenac (1\%), fentanyl (1\%), indomethacin $(1 \%)$, or morphine $(1 \%)$.

The most common doses of premedication were $37.5 \mathrm{mg} / 325 \mathrm{mg}$ of a combination of tramadol and acetaminophen $(29 \%), 5 \mathrm{mg}$ of diazepam (17\%), $50 \mathrm{mg}$ of tramadol (16\%), and $500 \mathrm{mg}$ of acetaminophen (12\%). The analysis of postoperative analgesia showed that $223 / 321$ (69\%) patients did not receive any analgesia after the operation. Among the $31 \%$ of patients who did receive analgesia or sedation 
after the operation, the most commonly used drugs were a combination of tramadol/acetaminophen (26\%), tramadol (23\%), and diazepam (18\%) (Table 1).

The most common doses for postoperative analgesic drugs were $37.5 \mathrm{mg} / 325 \mathrm{mg}$ of a combination of tramadol/ acetaminophen (28\%), $500 \mathrm{mg}$ of acetaminophen (18\%), and $5 \mathrm{mg}$ of diazepam (17\%). Patients who received analgesics most commonly received them within 3 days of the surgery (ranging from 1 to 9 days postimplantation). They received analgesics most commonly once (19\%) or twice (16\%) a day.

\section{Prospective study}

A total of 51 patients who underwent CED implantation from November 2014 to August 2015 were included in the prospective study. The highest recorded intensity of pain on the NRS was 8 during the procedure, 7 at 1 h postoperatively, and 6 at the remaining four postoperative measurements. The average postoperative pain at all time points was $<4$ (Figure 1 ). The

Table I Analgesia received after cardiac electronic device implantation in 321 patients

\begin{tabular}{ll}
\hline Postoperative analgesia & N (\%) \\
\hline Tramadol/acetaminophen & $31(26)$ \\
Tramadol & $28(23)$ \\
Diazepam & $22(18)$ \\
Acetaminophen & $21(17)$ \\
lbuprofen & $\mathrm{II}(9)$ \\
Methylphenobarbital & $2(2)$ \\
Diclofenac & $\mathrm{I}(\mathrm{I})$ \\
Fentanyl & $\mathrm{I}(\mathrm{I})$ \\
Indomethacin & $\mathrm{I}(\mathrm{I})$ \\
Metamizole & $\mathrm{I}(\mathrm{I})$ \\
\hline
\end{tabular}

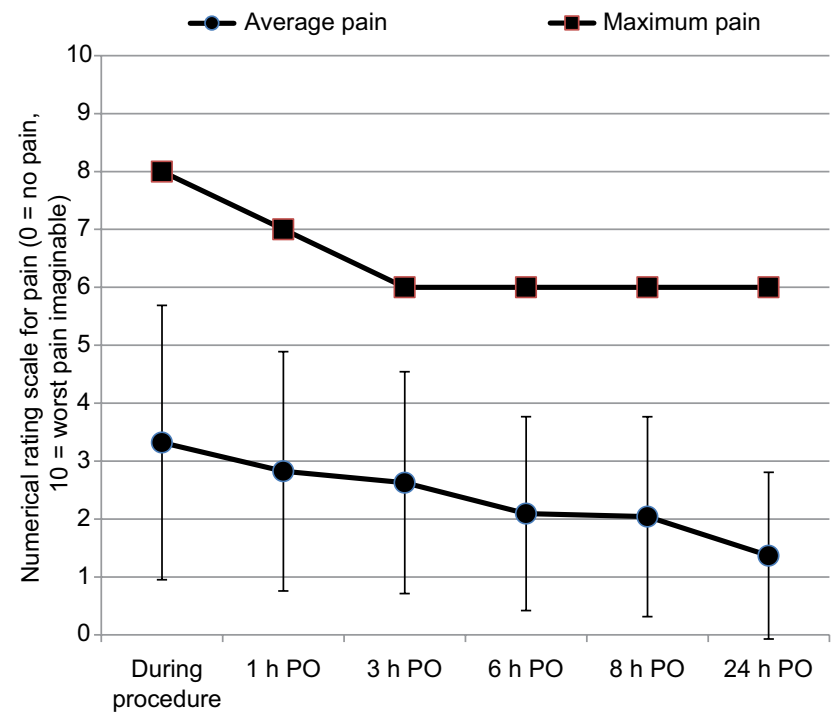

Figure I Maximum and average pain intensity during and after cardiac electronic device implantation (PO, postoperatively). Average pain presented as mean and standard deviation. most common locations of pain were the site of operation (44\%), shoulder (5\%), and chest (2\%) (Table 2).

\section{Comparison with guidelines for acute perioperative pain management}

Postoperative pain management during CED implantation at the study center was compared to the ASA guidelines for perioperative pain management. ${ }^{15}$ It was found that the study center did not adhere to the guidelines. Health workers involved with the postoperative care of patients were not trained by the anesthesiologists. Patients' medical notes did not contain information about previous pain intensity for any of the patients. Furthermore, the notes did not contain any information on any side effects of the drugs. Multimodal pain therapy was not used, and acetaminophen was rarely used as postoperative analgesia. Patients were not placed in separate rooms for postoperative recovery because CED implantation is considered a minor procedure in the day hospital, and there was no intensive patient follow-up.

\section{Discussion}

Our retrospective analysis indicated that patients undergoing CED implantation at the study center received inadequate perioperative pain management. It was found that $75 \%$ of patients did not receive any kind of premedication. All procedures were conducted under local anesthesia with lidocaine. Only $25 \%$ of patients received any analgesics or sedative drugs after the procedure. Furthermore, the prospective analysis showed that patients experienced severe pain during the procedure, with an intensity of up to 8 , indicating that anesthesia and analgesia were inadequate.

Guidelines for treating perioperative pain were published by the ASA in 2012..$^{15}$ The aim of the guidelines was to increase the efficacy and safety of acute perioperative pain management, to reduce the risk of side effects, to preserve patients' functions, and to increase patients' quality of life during the perioperative period. ${ }^{15}$ The guidelines emphasize the importance of preventing the development of side effects and chronic pain due to inappropriate analgesia. They also

Table 2 Location of the most intense pain in 51 patients undergoing cardiac electronic device implantation

\begin{tabular}{ll}
\hline Site & $\mathbf{N}(\%)$ \\
\hline Procedural site & $137(44)$ \\
Chest & $7(2)$ \\
Shoulder & $17(5)$ \\
Arm & $2(0.6)$ \\
Neck & $3(0.9)$ \\
No answer & $85(27)$ \\
\hline
\end{tabular}


emphasize education of the hospital staff by anesthesiologists. This training should include education about pain assessment and on pharmacological and nonpharmacological treatments for pain. According to the guidelines, anesthesiologists should regularly evaluate pain intensity, and the efficiency and side effects of therapy, and document these. Preoperative assessment of patients should include taking a detailed patient history, especially about previous pain, physical examination, and planning pain control considering the procedure that the patient is undergoing. ${ }^{15}$

A number of studies on the quality of postoperative pain management have been conducted in different countries to improve postoperative care and implement the guidelines. For example, a study conducted by Apfelbaum et $\mathrm{al}^{16}$ in the USA showed that $80 \%$ of patients experienced pain after the procedure. Among these, $86 \%$ had moderate, severe, or extremely severe pain which in many cases occurred after being discharged from hospital. ${ }^{16}$

Maier et $\mathrm{al}^{17}$ analyzed pain treatment in German hospitals during a 3-year period by interviewing patients undergoing surgical and nonsurgical procedures about their intensity of pain and efficiency of the treatment of pain. They found that $55 \%$ of patients from the surgical group and $57 \%$ from the nonsurgical group were not satisfied with their pain treatment. Furthermore, 39\% of nonsurgical and 15\% of surgical patients did not receive any analgesia even though they had pain. Pain therapy was considered inadequate for $46 \%$ of nonsurgical and $30 \%$ of surgical patients. The authors concluded that pain after surgical or nonsurgical procedures is still very common and its treatment is inadequate. ${ }^{17}$

A similar study conducted by Weiran et $\mathrm{al}^{18}$ in China indicated that postoperative pain was relieved within 3 days for $83 \%$ of patients. However, $20 \%$ of interviewed patients were not satisfied with their pain treatment, whereas $52 \%$ did not receive any analgesia even though $91 \%$ of patients reported pain. The authors concluded that, even though effective methods of pain treatment are available, there is a need for additional education of hospital staff, patients, and their families about pain treatment and also a need for better communication with patients in the postoperative period. ${ }^{18}$

The studies mentioned thus far did not relate specifically to CED implantation but to various other surgical procedures. However, Anderson et a ${ }^{19}$ recently presented a summary of their research conducted in London, UK, about analgesia after CED implantation at the 15th World Congress on Pain. These findings indicated that acetaminophen was given during the procedure only to patients who were under general anesthesia and that the same patients received opioids in
$43 \%$ of cases. Morphine was given to $83 \%$ of patients who underwent local anesthesia. They found that only $48 \%$ of patients received analgesia after discharge despite high pain intensity $(>6)$ but a year later, after educating the hospital staff, that number increased to $53 \%$. They concluded that pain after CED implantation is underrated and that hospital staff did not administer analgesia consistently. Even though educational programs helped to increase administration of analgesics after discharge, development of guidelines for pain treatment in cardiac device implantation was proposed. ${ }^{19}$

In this study, none of the patients received morphine after the procedure, which might indicate a lack of very severe pain among patients or a reluctance of physicians to administer morphine. In contrast to the study by Anderson et $\mathrm{al}^{19}$ in which $29 \%$ of patients underwent CED implantation under general anesthesia, all patients in the present study received local lidocaine anesthesia.

It was found that, at the study center in $2014,75 \%$ of patients did not receive any analgesic or sedative premedication, and $69 \%$ of patients did not receive any pain medication after the operation. As indicated by the high pain intensities reported during the procedure by patients in the prospective study, perioperative pain management was inadequate. It is possible that some patients received analgesics not for postoperative pain, but for painful comorbidities, considering the advanced age of the population. It is also possible that the patients are self-medicating and sharing analgesics between themselves to alleviate pain. It is known that sharing of even prescription analgesics is a common behavior that is viewed positively by patients and even some physicians. ${ }^{20,21}$

Comparing the practice at the study center to the ASA guidelines for perioperative pain management indicated lack of compliance with those guidelines. In particular, it was noted that there was no policy for acute pain management at the hospital. Very few studies of acute pain management procedures have been conducted in tertiary hospitals in Croatia. A study conducted in another department of the same hospital indicated inadequate perioperative pain management in patients undergoing complex ophthalmic procedures. ${ }^{14}$ Another study of chronic pain treatment in the same hospital showed long waiting times for referral to a tertiary pain clinic and for procedures and referrals to specialists. ${ }^{22}$ Understaffed pain clinics in Croatia and a narrow focus of work may explain their limited involvement in acute pain management. ${ }^{23}$ ASA guidelines recommend multimodal pain management, but this was not observed in the present study or the previous studies on acute perioperative pain management, ${ }^{14,24}$ and the treatment of chronic pain ${ }^{25}$ at the same hospital. 
Despite the ASA guidelines for acute perioperative pain treatment, ${ }^{15}$ it was found that anesthesiologists do not educate hospital staff on pain treatment. There is no routine evaluation of pain intensity, therapy efficiency, or side effects. Patients' medical records did not contain information about previous pain intensity or any side effects of the therapy. Even though CED implantation is a minor procedure, in most cases conducted under local anesthesia, and not requiring a long stay in the hospital, there is still a possibility of pain during the procedure and in the early postoperative period. Local guidelines for acute pain management should be developed, taking into account the complexity of the procedure and specific conditions of the setting.

These prospective data showed that patients experienced the most pain during the procedure, with some patients reporting severe pain (NRS score $>5$ ) in all postoperative measurements. Patients' pain reports during the procedure showed high variability, but pain is a highly subjective sensation; hence, this variability can be expected. Considering that almost every patient felt some postoperative pain, there is room for improving perioperative pain management related to CED implantation. Education of the hospital staff, in line with the ASA guidelines for perioperative pain management, ${ }^{15}$ could be the first step to remedy this situation.

Strengths of this study include its originality, since any previous studies on this subject could not be found. Furthermore, findings of this study are significant because this study identified the problem that no patients undergoing CED implantation had adequate pain management and pointed out the way to solve it by educating the staff and the introduction of acute pain management guidelines to alleviate suffering of patients and enable pain-free CED implantation procedures.

\section{Limitations}

Limitations of the study are its cross-sectional nature and relatively small number of patients involved in the prospective part because it was conducted in a single center; thus, it may not be representative of other centers, and there were some missing data on pain location. Further observational studies should explore pain intensity associated with CED implantation in terms of risk factors for pain development and factors related to the procedure (size of the device, extent of the surgical trauma, and duration of the procedure) and patient condition. Further studies on this subject should also include randomized controlled trials to assess the efficacy and safety of various analgesics and tranquilizers in the CED implantation setting as no such studies could be found in the medical literature.

\section{Conclusion}

All patients undergoing CED implantation at the study center received local anesthesia, and perioperative pain management was inadequate. Education of staff and introduction of acute pain management guidelines should be the next step to alleviate suffering of patients and enable pain-free CED implantation procedures.

\section{Acknowledgments}

The authors are grateful to all patients who participated in the study and to Dr Antonia Jelicic Kadic for critical reading of the manuscript. Many thanks to Prof Elizabeth Wager, of Sideview, Princes Risborough, for language editing; Sideview specializes in medical and academic publications.

\section{Disclosure}

The authors report no conflicts of interest in this work.

\section{References}

1. Herce B, Nazeyrollas P, Lesaffre F, et al. Risk factors for infection of implantable cardiac devices: data from a registry of 2496 patients. Europace. 2013;15(1):66-70.

2. Hill PE. Complications of permanent transvenous cardiac pacing: a 14-year review of all transvenous pacemakers inserted at one community hospital. Pacing Clin Electrophysiol. 1987;10(3 Pt 1):564-570.

3. Gadler F, Valzania C, Linde C. Current use of implantable electrical devices in Sweden: data from the Swedish pacemaker and implantable cardioverter-defibrillator registry. Europace. 2015;17(1):69-77.

4. Lohman D, Schleifer R, Amon JJ. Access to pain treatment as a human right. BMC Med. 2010;8:8.

5. Krnic D, Anic-Matic A, Dosenovic S, Zezelic S, Draganic P, Puljak L. National consumption of opioid and nonopioid analgesics in Croatia: 2007-2013. Ther Clin Risk Manag. 2015;11:1305-1314.

6. Hamunen K, Laitinen-Parkkonen P, Paakkari P, et al. What do different databases tell about the use of opioids in seven European countries in 2002? Eur J Pain. 2008;12(6):705-715.

7. De Conno F, Ripamonti C, Brunelli C. Opioid purchases and expenditure in nine western European countries: "are we killing off morphine?" Palliat Med. 2005;19(3):179-184.

8. Lasinkas M. Trends in the Consumption of Analgesic Drugs in Lithuania iz 2005-2007 [master's thesis]. Kauno Medicinos Universitetas. Available from: http://vddb.library.lt/obj/LT-eLABa-0001:E.02 200 8 D_20080616_100523-46119. Accessed December 3, 2008.

9. Gudin JA. The changing landscape of opioid prescribing: long-acting and extended-release opioid class-wide Risk Evaluation and Mitigation Strategy. Ther Clin Risk Manag. 2012;8:209-217.

10. Awan H, Durrani Z. Postoperative pain management in the surgical wards of a tertiary care hospital in Peshawar. J Pak Med Assoc. 2015;65(4):358-361.

11. Sansone P, Pace MC, Passavanti MB, Pota V, Colella U, Aurilio C. Epidemiology and incidence of acute and chronic post-surgical pain. Ann Ital Chir. 2015;86:285-292.

12. Joris JL, Georges MJ, Medjahed K, et al. Prevalence, characteristics and risk factors of chronic postsurgical pain after laparoscopic colorectal surgery: retrospective analysis. Eur J Anaesthesiol. 2015;32(10):712-717.

13. Lesin M, Domazet Bugarin J, Puljak L. Factors associated with postoperative pain and analgesic consumption in ophthalmic surgery: a systematic review. Surv Ophthalmol. 2015;60(3):196-203.

14. Lesin M, Sundov ZD, Jukic M, Puljak L. Postoperative pain in complex ophthalmic surgical procedures: comparing practice with guidelines. Pain Med. 2014;15(6):1036-1042. 
15. Thompson M, Tiwari A, Fu R, Moe E, Buckley DI. A Framework to Facilitate the Use of Systematic Reviews and Meta-analyses in the Design of Primary Research Studies. [Prepared by the Oregon Evidencebased Practice Center under Contract HHSA 290-2007-10057-I]. Rockville, MD: Agency for Healthcare Research and Quality; 2012. AHRQ Publication No. 12-EHC009-EF. Available from: https://www. ncbi.nlm.nih.gov/pubmedhealth/PMH0076923/. Accessed March 29, 2017.

16. Apfelbaum JL, Chen C, Mehta SS, Gan TJ. Postoperative pain experience: results from a national survey suggest postoperative pain continues to be undermanaged. Anesth Analg. 2003;97(2):534-540.

17. Maier C, Nestler N, Richter $\mathrm{H}$, et al. The quality of pain management in German hospitals. Dtsch Arztebl Int. 2010;107(36):607-614.

18. Weiran L, Lei Z, Woo SM, et al. A study of patient experience and perception regarding postoperative pain management in Chinese hospitals. Patient Prefer Adherence. 2013;7:1157-1162.

19. Anderson RH, Cox FJ, Jaggar SI. Analgesia provision for cardiac device implantation - an unrecognised need. 15th World Congress on Pain. October 6-11, 2014. Buenos Aires, Argentina; 2014. Available from: http://docplayer.net/44253580-15th-world-congress-on-pain.html. Accessed March 29, 2017.
20. Markotic F, Puljak L. Risks associated with borrowing and sharing of prescription analgesics among patients observed by pain management physicians in Croatia: a qualitative study. J Pain Res. 2016;9: 1143-1151.

21. Markotic F, Vrdoljak D, Puljiz M, Puljak L. Risk perception about medication sharing among patients: a focus group qualitative study on borrowing and lending of prescription analgesics. J Pain Res. 2017; 10:365-374.

22. Triva P, Jukic M, Puljak L. Access to public healthcare services and waiting times for patients with chronic nonmalignant pain: feedback from a tertiary pain clinic. Acta Clin Croatica. 2013;52(1):79-85.

23. Fidahic M, Dogan K, Sapunar D, Puljak L. National survey of pain clinics in Croatia: organization and services. Acta Med Acad. 2015;44(1): $18-30$.

24. Boric K, Boric M, Boric T, Puljak L. Analysis of perioperative pain management in vascular surgery indicates that practice does not adhere with guidelines: a retrospective cross-sectional study. J Pain Res. 2017; 10:203-209.

25. Jukic M, Kardum G, Sapunar D, Puljak L. Treatment of chronic musculoskeletal back pain in a tertiary care pain clinic. J Musculoskelet Pain. 2012;20(4):277-283.
Journal of Pain Research

\section{Publish your work in this journal}

The Journal of Pain Research is an international, peer reviewed, open access, online journal that welcomes laboratory and clinical findings in the fields of pain research and the prevention and management of pain. Original research, reviews, symposium reports, hypothesis formation and commentaries are all considered for publication.

\section{Dovepress}

The manuscript management system is completely online and includes a very quick and fair peer-review system, which is all easy to use. Visit http://www.dovepress.com/testimonials.php to read real quotes from published authors. 